\title{
Fördern realitätsbasierte UIs kollaborative Rechercheaktivitäten?
}

\author{
Mathias Heilig, Mischa Demarmels, Katrin Allmendinger, Jens Gerken, \\ Harald Reiterer \\ Arbeitsgruppe Mensch-Computer Interaktion, Universität Konstanz
}

\section{Zusammenfassung}

Soziale Aktivitäten sind während eines Rechercheprozesses von essentieller Bedeutung, werden jedoch bisher in aktuellen digitalen Recherchesystemen nur unzureichend berücksichtigt. Social, Tangible und Surface Computing bieten erste Ansätze zur Lösung des Problems. Darauf aufbauend stellt dieser Beitrag drei verschiedene Benutzerschnittstellen für die Aktivität des gemeinsamen Filterns und Explorierens vor. Dabei wurden die Benutzerschnittstellen bewusst mit unterschiedlichen Interaktionstechniken umgesetzt, von sehr realitätsbasiert über rein virtuell bis hin zu einem eher klassischen Ansatz. Weiterhin werden Ergebnisse einer Evaluationsstudie vorgestellt, in der insbesondere die Auswirkungen der verschiedenen Ansätze auf das kollaborative Arbeiten untersucht wurden.

\section{$1 \quad$ Einleitung}

Viele theoretische Modelle, die sich mit Informationssuche beschäftigen (z. B. Kuhlthau 2004), stellen die essenzielle Bedeutung sozialer Interaktion während eines Rechercheprozesses heraus. Doch in heutigen digitalen Recherchesystemen, wie sie beispielsweise häufig in Bibliotheken zu finden sind, wird gerade dieser Aspekt wenig beachtet. Ein Grund dafür sind die Limitierungen der verwendeten Desktop oder Terminal PCs, die mit Maus und Tastatur gesteuert werden, welche das gemeinsame Arbeiten und Recherchieren nur bedingt ermöglichen (Amershi \& Morris 2008). Ein Ansatz zur Lösung dieses Problems ist die Aufteilung der gemeinsamen Nutzung auf mehrere Endgeräte (vgl. Hoare \& Sorensen 2006). Durch die Übertragung der sozialen Interaktion aus der realen Welt in die digitale Welt gehen jedoch entscheidende Aspekte verloren, wie z. B. Blickkontakt, Gesten und Körperhaltung während einer Diskussion. Einen anderen Ansatz stellen so genannte Multitouch-Tische dar. Der hierbei verwendete horizontale Formfaktor und die Möglichkeit der gleichzeitigen Bedienung per Berührung, demokratisieren die Interaktion und unterstützen somit die Kollaboration, wie in verschiedenen Forschungsarbeiten bereits gezeigt wurde (vgl. Morris et al. 2010). Eine in der Mensch-Computer Interaktion zunehmend beachtete Theorie, die diesen Schluss nahe legt, ist die aus den Kognitionswissenschaften bekannte Embodiment-Theorie 
(Gibbs 2006). Sie legt dar, dass die kognitive Entwicklung ganz maßgeblich durch die körperliche und soziale Interaktion mit Objekten bzw. Lebewesen in unserer Umwelt beeinflusst wird. Das Schlagwort Reality-Based Interaction (Jacob et al. 2008) fasst die oben genannten Erkenntnisse, die technischen Entwicklungen auf den Gebieten der multimodalen Interaktion sowie des Tangible und Social Computing zu einem neuen Paradigma zusammen. Generelles Ziel dabei ist es, die Interaktion mit dem Computer an der Interaktion mit der realen, nicht digitalen Welt zu orientieren und so realitätsbezogener und dadurch natürlicher zu gestalten. Bezogen auf den Rechercheprozess ist es daher entscheidend, Werkzeuge zu entwickeln, welche die Fähigkeiten des Menschen unter Verwendung von passenden Technologien optimal unterstützen. Ziel ist es demnach eine Vermischung (engl. Blending) von realitätsbezogener Interaktion mit den virtuellen Recherchefähigkeiten des Computers zu erreichen. Dieser Beitrag stellt drei Werkzeuge für Rechercheaktivitäten vor, welche diese Vermischung in unterschiedlichen Ausprägungen realisieren (vgl. Abbildung 3: physisches Such-Token, virtuelles Suchwerkzeug und klassische Eingabemaske). Die Werkzeuge fokussieren dabei auf die Aktivität des Explorierens und Filterns von Rechercheergebnissen. In einer Evaluationsstudie wurde überprüft, welche Unterschiede sich aus den verschiedenen Designansätzen bei einer gemeinsamen Exploration von Rechercheergebnissen ergeben.

2 Grundlagen
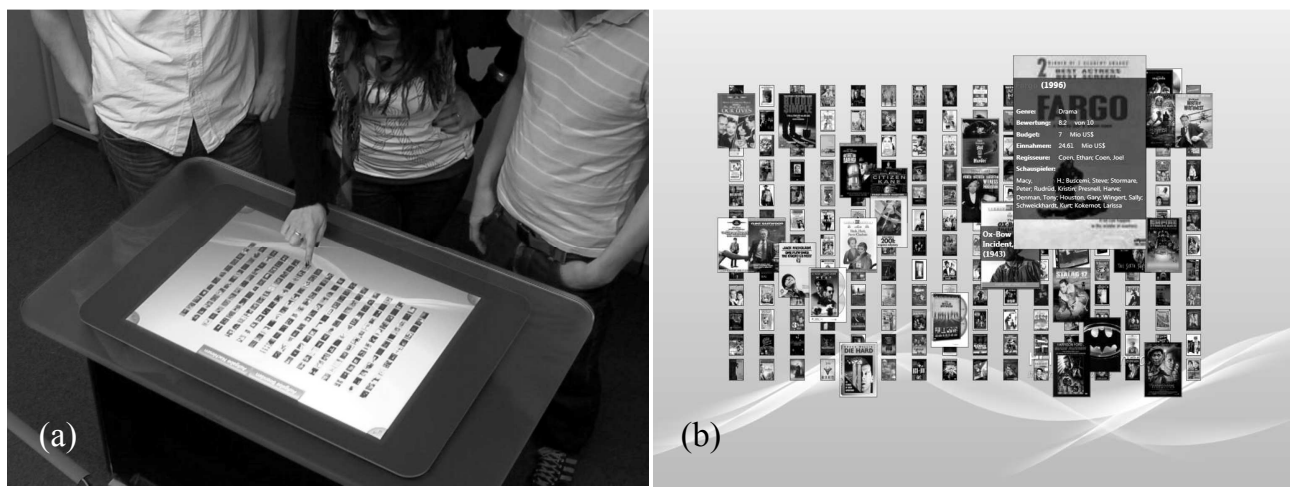

Abbildung 1: (a) Der Multitouch-Tisch während der Evaluationsstudie mit 3 kollaborativ arbeitenden Probanden. (b) Durch die Definition von Filterkriterien erfahren die relevanten Filmobjekte in der Visualisierung eine Vergrößerung. Durch den gewonnenen Bildschirmplatz können mehr Informationen angezeigt werden.

Wie oben beschrieben, ermöglichen Multitouch-Displays eine gleichberechtigte Form der Kommunikation, da mehrere Benutzer die Möglichkeit haben simultan zu interagieren und somit gewohnte soziale Konventionen unmittelbar Berücksichtigung finden. Deshalb wird als Basis der Entwicklung der verschiedenen Werkzeuge ein Multitouch-Tisch genutzt (vgl. Abbildung 1a). Für die Untersuchung der Werkzeuge wird eine speziell entwickelte Visualisierung verwendet, die knapp 200 Kinofilme mit verschiedenen Metadaten (z. B. Titel, Genre und Schauspieler) sowie deren Filmposter darstellt. Die Visualisierung stellt die einzelnen 
Filme als virtuelle Objekte in einem Gitter angeordnet auf dem Multitouch-Tisch dar (vgl. Abbildung 1a). Die Filmobjekte werden dabei in der Ausgangskonfiguration durch ihr Filmposter dargestellt. Um die Einflüsse auf die Benutzerstudie so gering wie möglich zu halten, sind die Interaktionsmöglichkeiten mit der Visualisierung bewusst sehr beschränkt. So wird beispielsweise auf ein mögliches Zooming der einzelnen Filmobjekte oder der gesamten Visualisierung verzichtet.

Die in folgendem beschriebenen Funktionalitäten (Sensitivität und gewichtete Bool'sche Verknüpfungen) wurden explizit für die Evaluation ausgewählt, da beide Methoden das Erlebnis des kollaborativen Explorierens und Filterns in einer Gruppe fördern.

Sensitivität: Um es mehreren Benutzer gleichzeitig zu ermöglichen mit einer Menge von Informationsobjekten zu arbeiten, ist es notwendig, dass die Aktionen der anderen Gruppenmitglieder nachvollzogen werden können. Übliche Ansätze zur Recherche verfolgen die Strategie beim Filtern nicht relevante Informationsobjekte auszublenden. Wenn jedoch mehrere Personen eine Datenmenge explorieren, kann ein komplettes Ausblenden der nicht relevanten Informationsobjekte - vor allem beim simultanen Definieren mehrerer Filterkriterien - zu Verwirrung führen. Deswegen wird für die Werkzeuge eine andere Methode verwendet, welche sich an das Konzept der "Sensitivität“ (Tweedy et al. 1994) anlehnt. Stimmt ein definiertes Filterkriterium mit einem Metadatum überein, so wird der Film vergrößert und setzt sich dadurch von den restlichen Filmobjekten ab (vgl. Abbildung 1b). Datenobjekte, die nicht auf ein Filterkriterium zutreffen, werden nicht komplett aus der Ergebnisdarstellung entfernt, sondern verkleinert und dadurch in den Hintergrund gerückt. Durch die Vergrößerung der Filmobjekte wird außerdem je nach Bildschirmplatz eine in der Granularität der Information variierende Darstellung für ein Objekt verwendet (vgl. „semantic zooming“, Perlin \& Fox 1993). Je mehr Pixel ein Filmobjekt zur Verfügung hat, desto mehr Detailinformationen werden angezeigt (vgl. Abbildung 1b). In den sichtbaren Detailinformationen werden zusätzlich die relevanten Filterkriterien durch eine Einfärbung hervorgehoben.

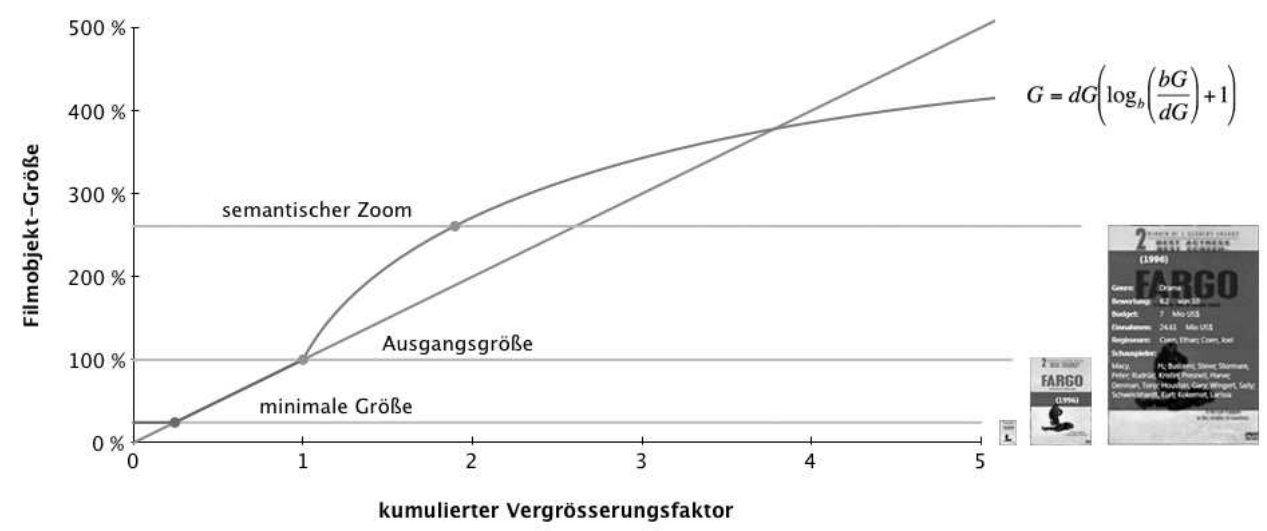

Abbildung 2: Das Diagram zeigt die Vergrößerungsfunktion der Filmobjekte abhängig vom kumulierten Vergrößerungsfaktor. $d G$ stellt die Ausgangsgröße eines nicht gefilterten Filmobjektes dar, $b G$ die durch die Überlagerung von mehreren Filtern berechnete Größe des aktuellen Filmobjektes und b die Basis des Logarithmus, welche je nach Ausgangslage angepasst werden kann. 
Gewichtete Bool'sche Verknüpfungen: Die Verknüpfung von unterschiedlichen Filterkriterien stellt ein wesentliches Merkmal zur Unterstützung des kollaborativen Rechercheerlebnises dar. Es ermöglicht den Teilnehmern, sich individuell in den Rechercheprozess einzubringen. Die vorgestellten Werkzeuge verwenden hierzu eine „UND“ Verknüpfung im Sinne der Bool'schen Logik. Dadurch werden Filmobjekte, die auf mehrere Kriterien zutreffen nach dem Konzept der Sensitivität größer dargestellt als Filmobjekte, die nur ein Kriterium erfüllen. Darüber hinaus ist eine Gewichtung jedes einzelnen Filterkriteriums möglich. Die Benutzer haben dadurch beispielsweise die Möglichkeit, in einer Diskussion das Gewicht eines Filterkriteriums interaktiv zu erhöhen, um somit anderen Diskussionsteilnehmern einen Aspekt zu kommunizieren.

Das Gewicht jedes einzelnen Filterkriteriums kann durch die Benutzer auf einer kontinuierlichen Skala (von 0 bis 2) verändert werden. Das mathematische Modell hinter der Gewichtung von Filterkriterien ist an das Konzept der gewichteten Bool'schen Verknüpfungen von Waller \& Kraft (1979) angelehnt. Je höher das Gewicht (zwischen 1 und 2) gewählt wird, desto mehr Einfluss hat ein Filter auf die Größe relevanter Filmobjekte. Bool'sche 'NICHT' Verknüpfungen können dagegen durch ein niedriges Gewicht (zwischen 0 und 1) ausgedrückt werden. Alle Einflüsse der verschiedenen Filter auf ein bestimmtes Filmobjekt werden zu der Größe dieses Objektes hinzu multipliziert (vgl. Abbildung 2, blaue Gerade). Danach wird eine logarithmische Anpassung der Größe der Filmobjekte auf der Visualisierung ausgeführt. Die Größe eines Objektes $(G)$ berechnet sich nach der in Abbildung 2 hinterlegten Funktion in Echtzeit. Damit die vergrößerten Filmobjekte schneller genügend Platz zum Anzeigen von weiteren Detailinformationen haben, werden Vergrößerungsfaktoren über dem Wert 1 in einer ersten Phase verstärkt (vgl. Abbildung 2, grüne Kurve). Sobald alle Detailinformationen $\mathrm{zu}$ einem Filmobjekt sichtbar sind, wird der Faktor in einer zweiten Phase stetig abgeschwächt und verläuft schließlich unter der blauen, linearen Kurve. Dadurch wird verhindert, dass sehr hoch gewichtete Filmobjekte zu viel des Bildschirmplatzes einnehmen und dadurch viele andere Filmobjekte überdecken. Filme die durch den kumulierten Vergrößerungsfaktor eine Verkleinerung erfahren, werden bis zu einer vordefinierten Mindestgröße linear verkleinert (vgl. Abbildung 2, rote Kurve).

\section{$3 \quad$ Vorstellung der Werkzeuge}

Im Folgenden werden drei verschiedene Werkzeuge (vgl. Abbildung 3) für kollaborative Rechercheaktivitäten auf Basis der oben beschriebenen Grundlagen vorgestellt. Dabei handelt es sich um (a) ein physisches Such-Token, (b) ein virtuelles Suchwerkzeug und (c) eine klassische Eingabemaske. Die im vorangegangen Abschnitt motivierten Grundfunktionalitäten sind für alle drei Werkzeuge mit den verschiedenen Designausprägungen konsequent umgesetzt, jedoch variiert der Grad an realitätsbasierter Interaktion. Ziel der Gestaltung war es, unterschiedliche Ausprägungen in der Realitätsbezogenheit der Interaktion zu finden, um deren Einfluss auf das kollaborative Verhalten der Nutzer untersuchen zu können. 

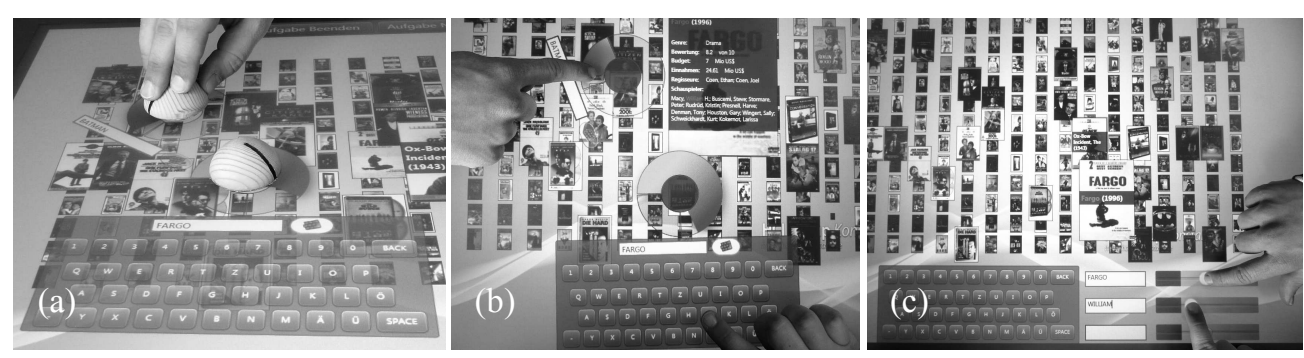

Abbildung 3: Alternative Werkzeuge (a) physisches Such-Token (b) virtuelles Suchwerkzeug und (c) klassische Eingabe Maske.

\subsection{Physisches Such-Token}

Mit den Such-Tokens (vgl. Abbildung 3a) wird eine (be-)greifbare Benutzerschnittstelle (eng. Tangible User Interface, kurz TUI) als kollaboratives Filterwerkzeug vorgestellt, das bewusst sehr realitätsbezogen entworfen wurde. TUIs wurden bereits 1997 von Ishii und Ullmer zur Anwendung im Kontext von Informationsmanagement, -verarbeitung und -manipulation vorgeschlagen. Ein Beispiel dafür stellen die „Tangible Query Interfaces“ von Ullmer et al. (2003) dar. Dabei handelt es sich um Konzepte, die für einen einzelnen Benutzer ausgelegt sind. TUIs sind jedoch durch ihren inhärenten physischen Aufforderungscharakter (engl. Affordance), laut Ishii und Ullmer (1997) in Gruppensituation besser sichtbar und nachvollziehbar als digitale Benutzerschnittstellen. Auch kann durch die gleichzeitige Verwendung mehrerer Such-Tokens eine Mehrbenutzerinteraktion leicht umgesetzt werden (Blackwell et al. 2004).

Das in diesem Beitrag vorgestellte Konzept der Such-Tokens sieht vor, physische Drehknöpfe auf dem Multitouch-Tisch als ,on-screen controls' zu verwenden, angelehnt an die Arbeit von Weiss et al. (2009). Jedes Such-Token auf dem Multitouch-Tisch verkörpert dabei einen benutzerdefinierten Such- bzw. Filterbegriff. Beim Auflegen eines Such-Tokens auf dem Multitouch-Tisch erscheint eine Visualisierung, welche virtuell mit dem physischen Token verbunden ist. Die Visualisierung setzt sich aus drei Teilen zusammen: Einem Texteingabefeld für die Filterbegriffe, einer virtuellen Tastatur, um die Begriffe einzugeben, sowie einer ringförmigen Skala, um die Gewichtung darzustellen. Wird das Such-Token auf dem Multitouch-Tisch verschoben oder gedreht, so verhält sich die virtuelle Darstellung analog zur realen Bewegung. Durch diese Möglichkeit der Ausrichtung kann das Werkzeug von allen Seiten des Tisches aus bedient werden. Außerdem bietet ein physisches Objekt weitere Mehrwerte, die aus der realen Welt bekannt sind. Beispielsweise kann eine Aufforderung an ein Teammitglied, sich an der Recherche aktiv zu beteiligen, durch das Übergeben eines realen Token ausgedrückt werden.

Nachdem ein Filterkriterium eingegeben wurde, kann die Tastatur der Such-Token Visualisierung durch eine Tap-Geste auf das Eingabefeld ausgeblendet werden. Daraufhin funktioniert das Such-Token wie ein Drehknopf, vergleichbar mit dem einer Stereo-Anlage oder einem Licht-Dimmer. Durch Drehen des Such-Token kann die Gewichtung des Filterkriteriums verändert werden. Wird es nach rechts gedreht, so steigert sich das Gewicht des Filter- 
kriteriums und somit die Größe der relevanten Filmobjekte. Das Drehen des Such-Token nach links verringert das Gewicht und damit simultan die Größe treffender Filmobjekte. Die virtuell dargestellte Skala um das physische Such-Token zeigt die aktuelle Gewichtung des Filterkriteriums durch einen Farbverlauf an (vgl. Abbildung 3a). Wird ein Such-Token vom Tisch weggenommen, so wird die dazugehörige digitale Visualisierung ausgeblendet und das entsprechende Filterkriterium zurückgesetzt.

\subsection{Virtuelles Suchwerkzeug}

Das virtuelle Suchwerkzeug (vgl. Abbildung 3b) bildet die Funktionen des physischen SuchToken konsequent digital auf dem Multitouch-Tisch ab. Es wurde entwickelt, um die Unterschiede zwischen physischer und virtueller Ausprägung in der späteren Evaluation festzustellen. Durch Tippen in eine der gekennzeichneten Ecken des Multitouch-Tisches können virtuelle Suchwerkzeuge erzeugt werden. Sie werden durch Visualisierungen verkörpert, welche die drei Elemente der Visualisierung der physischen Such-Token aufgreifen: Texteingabefeld, virtuelle Tastatur und ringförmige Skala. Das Werkzeug kann per Drag-Geste über den Bildschirm bewegt und mittels einer (Zwei-Finger)Dreh-Geste rotiert werden. Durch Ziehen des Werkzeuges über den Bildschirmrand, kann es wieder vom Bildschirm entfernt werden und der Filter wird gelöscht. Die Einstellung der Gewichtung geschieht per Fingergeste direkt auf der ringförmigen Skala, ähnlich dem Konzept eines „iPod Click Wheels“.

\subsection{Klassische Eingabemaske}

Die klassische Eingabemaske (vgl. Abbildung 3c) wurde zum Vergleich der zwei oben genannten Konzepte bewusst im Kontrast mit einem konservativen Ansatz und wenig realitätsbasierten Charakteristiken geschaffen. Sie besteht aus einer einzigen virtuellen Tastatur, drei Texteingabefeldern sowie drei dazugehörigen berührungsempfindlichen Slidern. Die drei Texteingabefelder ergeben sich aus dem Aufbau der Evaluationsstudie, die mit jeweils 3 Personen gleichzeitig durchgeführt wurde. In jedes Texteingabefeld kann jeweils ein Filterkriterium über die virtuelle Tastatur eingegeben werden. Die Gewichtung der Filterkriterien wird über die Slider eingestellt. Die drei Slider sind, den anderen Werkzeugen entsprechend, von mehreren Personen gleichzeitig bedienbar. Die gesamte Eingabemaske ist fest auf dem Bildschirm verortet. Während der Untersuchung hatten die Versuchspersonen jedoch die Möglichkeit die Positionen um den Tisch zu verändern. Da feste Positionen vermieden werden sollten, wurde die Evaluationsstudie im Stehen durchgeführt.

\section{$4 \quad$ Evaluation}

Um festzustellen inwiefern die verschiedenen entwickelten Werkzeuge das Arbeiten und das Verhalten in kollaborativen Situationen beeinflussen, wurde eine vergleichende Evaluationsstudie durchgeführt. Von besonderem Interesse ist dabei, wie sich die gegensätzlichen Designansätze, von den sehr realitätsbezogenen Such-Tokens, über die rein virtuellen Such- 
werkzeuge, bis hin zu der eher klassischen Eingabemaske unterscheiden und mit welchem Ansatz das kollaborative Filtern und Explorieren am besten unterstützt werden kann.

An der Untersuchung nahmen 18 studentische Versuchspersonen teil. Das Durchschnittsalter liegt bei 23,5 Jahren $(\mathrm{SD}=3,9)$, 56 Prozent der Teilnehmer sind männlich. Vor der Versuchsdurchführung füllten die Probanden einen Fragebogen zur Erfassung der Mediennutzung aus. 89 Prozent der Teilnehmer haben bereits Erfahrung mit berührungssensitiven Bildschirmen gesammelt, jedoch nur 50 Prozent der Teilnehmer haben bereits mittels TUIs mit einem Computer interagiert. Die Versuchspersonen wurden in 3-Personen-Gruppen (Triaden) eingeteilt. Die 6 Triaden durchliefen alle 3 Nutzungsbedingungen physisches SuchToken, virtuelles Suchwerkzeug und klassische Eingabemaske, weshalb bei der statistischen Auswertung Meßwiederholungsanalysen verwendet werden (Bortz \& Döring 2006). Die Reihenfolge der Werkzeuge wurde dabei vollständig ausbalanciert.

Nach dem Fragebogen zur Mediennutzung explorierten die Triaden die Bedienung des berührungssensitiven Tisches und des jeweils ersten Werkzeuges zunächst selbständig. Anschließend erfolgte eine systematische Instruktion durch den Versuchsleiter, um eine einheitliche Wissensbasis zwischen allen Triaden zu garantieren. Daraufhin folgte eine Übungsphase mit Probeaufgaben, in der überprüft wurde, inwieweit die Probanden die Instruktionen verstanden hatten. In der sich anschließenden Versuchsphase wurden pro Werkzeug je 4 Aufgaben bearbeitet. Der Grad der Aufgabenschwierigkeit wurde hierbei gesteigert und begann bei relativ einfachen Suchaufgaben (z. B. „Wie viele Filme, an denen ,Spielberg' mitgewirkt hat, befinden sich im Datenbestand?") und steigerte sich dann zu komplexeren Aufgaben (z. B. „Welches ist der am besten bewertete Film aus dem Genre Drama an dem die Person/en mit dem Namen ,Coen' mitgewirkt hat/haben?“). Zur Lösung der Aufgaben waren Konsensentscheidungen innerhalb der Gruppe zu treffen und das Ergebnis musste anschließend in ein Formular eingegeben werden. Nach Bearbeitung der Aufgaben wurde von den Teilnehmern ein Fragebogen zur Beurteilung der Werkzeuge individuell ausgefüllt. Dieser Ablauf wurde für jedes der Werkzeuge wiederholt. Nach der Aufgabenphase wurde ein Fragebogen ausgeteilt, in dem alle drei Werkzeuge hinsichtlich unterschiedlicher Aspekte (z. B. Natürlichkeit der Bedienung) von den Probanden verglichen werden sollten. Am Ende wurden in einem Gruppeninterview offene Fragen zu den Werkzeugen gestellt und die Möglichkeit zur Diskussion geboten. Die Dauer der Studie betrug für die Versuchspersonen 1 bis 1,5 Stunden. Sie erhielten 5 Euro als Aufwandsentschädigung. Die Versuchsdaten wurden zum Teil über die bereits beschriebenen Fragebögen und zum Teil über Beobachtung erhoben.

Die per Video aufgezeichneten Interaktionen der Triaden wurden im Nachhinein von 3 Beobachtern ausgewertet, die jeweils einen Probanden beobachteten. Es wurde in per Metronom vorgegebenen 5-Sekunden-Intervallen erfasst, ob die Versuchspersonen mit den Werkzeugen bzw. der Benutzerschnittstelle interagierten oder nicht. Die Anzahl der Interaktionen wurden durch die Anzahl der 5-Sekunden Intervalle dividiert, um eine Prozentaussage unter Berücksichtigung der Versuchszeit zu erzielen (vgl. Abbildung 4).

Im Folgenden wird zunächst beschrieben wie stark die Versuchspersonen mit den verschiedenen Werkzeugen und der Visualisierung interagiert haben. Danach wird auf die Fragebogenergebnisse hinsichtlich der Nutzung der einzelnen Werkzeuge eingegangen. 


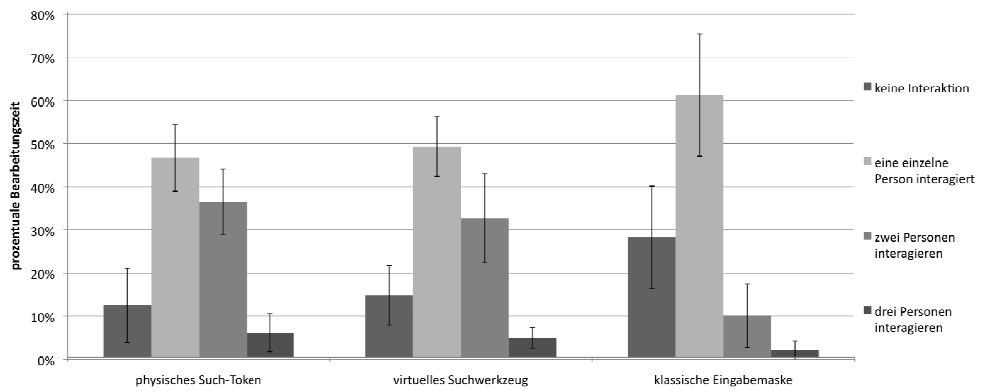

Abbildung 4: Nutzungsverhalten hinsichtlich der Werkzeuge nach prozentualer Bearbeitungszeit der Aufgaben

Abbildung 4 ist zu entnehmen, dass die Versuchspersonen die Möglichkeit, der parallelen Interaktion mit der Benutzerschnittstelle im Falle des virtuellen Suchwerkzeugs und des physischen Such-Token genutzt haben. Während unter der Nutzungsbedingung klassische Eingabemaske lediglich 10 Prozent der Zeit zwei Personen parallel interagiert haben, wurde bei dem virtuellen Suchwerkzeug 32 Prozent und bei dem Such-Token 36 Prozent der Zeit parallel von zwei Personen mit dem Interface interagiert. Eine gemeinsame Interaktion aller drei Probanden konnte bei allen Werkzeugen nur vereinzelt beobachtet werden. Umgekehrt erfolgte die Interaktion mit dem Werkzeug durch nur eine Person der Triade in der Nutzungsbedingung klassische Eingabemaske mit 61 Prozent am häufigsten im Vergleich zu virtuellem Suchwerkzeug mit 49 Prozent und physischem Such-Token mit 46 Prozent.

\begin{tabular}{|l|c|c|c|}
\hline & $\begin{array}{l}\text { Frage 1: Geeignet für } \\
\text { koll. Recherche? }\end{array}$ & $\begin{array}{l}\text { Frage 2: Persönlich am } \\
\text { meisten zugesagt? }\end{array}$ & $\begin{array}{l}\text { Frage 3: Am anstren- } \\
\text { gendsten zu Bedienen? }\end{array}$ \\
\hline Physisches Such-Token & $59 \%$ & $47 \%$ & $22 \%$ \\
\hline Virtuelles Suchwerkzeug & $41 \%$ & $24 \%$ & $56 \%$ \\
\hline Klassische Eingabemaske & $0 \%$ & $29 \%$ & $22 \%$ \\
\hline
\end{tabular}

Tabelle 1: Ergebnisse des vergleichenden Fragebogen

Aus den subjektiven Antworten der Fragebögen, ergab sich ein ähnliches Bild. Im Durchschnitt empfanden die Probanden die Bedienung der klassischen Eingabemaske am wenigsten geeignet für kollaborative Recherche $(\mathrm{M}=3.06, \mathrm{SD}=1.55$; Skala von 1 ,überhaupt nicht geeignet" bis 7 ,sehr gut geeignet") im Vergleich zu den physischen Such-Tokens $(\mathrm{M}=5.78$, $\mathrm{SD}=1.43)$ und den virtuellen Suchwerkzeugen $(\mathrm{M}=5.50, \mathrm{SD}=1.25)$. Die Unterschiede sind statistisch signifikant (klassische Eingabemaske versus virtuelles Suchwerkzeug: $\mathrm{t}(17)=$ -6.27, p < 0.01; klassische Eingabemaske versus physisches Such-Token: $\mathrm{t}(17)=-6.50, \mathrm{p}<$ 0.01). Die Bewertung der physischen Such-Tokens und der virtuellen Suchwerkzeuge unterscheidet sich nicht signifikant $(\mathrm{t}(17)=-1.05, \mathrm{p}>0.1)$. Auf die Frage nach der Aktivität der anderen Teammitglieder gaben die Probanden die niedrigste Aktivität in der Nutzungsbedingung Eingabemaske ( $\mathrm{M}=5.39, \mathrm{SD}=0.98$; Skala von 1 „,sehr passiv“ bis 7 ,sehr aktiv“) im 
Vergleich zu dem physischen Such-Token $(\mathrm{M}=6.00, \mathrm{SD}=0.84)$ und dem virtuellen Suchwerkzeug $(\mathrm{M}=5.89, \mathrm{SD}=0.83)$ an. Die Unterschiede sind signifikant (klassische Eingabemaske versus physischem Such-Token: $\mathrm{t}(17)=-2.65, \mathrm{p}<0.05)$ bzw. knapp nicht signifikant (klassische Eingabemaske versus virtuellem Suchwerkzeug: $\mathrm{t}(17)=-2.03, \mathrm{p}<0.1)$. Auch hier ist zwischen den physischen Such-Tokens und den virtuellen Suchwerkzeugen kein signifikanter Unterschied festzustellen $(\mathrm{t}(17)=-0.81, \mathrm{p}>0.1)$.

Die subjektive Einschätzung der Werkzeuge, welche durch den vergleichenden Fragebogen ermittelt wurde, stellt die Unterschiede zwischen den physischen Such-Tokens und den virtuellen Suchwerkzeugen heraus. 59 Prozent der Teilnehmer gaben an, dass das Werkzeug physischer Such-Token ihrer Meinung nach am besten für gemeinsames Recherchieren geeignet sei, für das virtuelle Suchwerkzeug entschieden sich 41 Prozent der Teilnehmer, für die klassische Eingabemaske 0 Prozent (vgl. Tabelle 1, Frage 1; ein Teilnehmer hatte 2 Alternativen ausgekreuzt und wurde nicht in die Auswertung einbezogen). Den Probanden hat insgesamt das physische Such-Token am meisten zugesagt, Rang $2 \mathrm{nahm}$ im direkten Vergleich die klassische Eingabemaske ein und Rang 3 das virtuelle Suchwerkzeug (vgl. Tabelle 1, Frage 2). Des Weiteren wurden die Probanden gebeten einzuschätzen, wie anstrengend die Bedienung der Werkzeuge gewesen sei (vgl. Tabelle 1, Frage 3): es ergibt sich ein Ergebnismuster, das für die beiden Bedingungen klassische Eingabemaske und physisches Such-Token spricht. Während 56 Prozent der Teilnehmer das virtuelle Suchwerkzeug als am anstrengendsten in der Bedienung beurteilten, entfielen nur je 22 Prozent der Antworten auf die klassische Eingabemaske und das Such-Token.

\section{$5 \quad$ Diskussion und Ausblick}

Abschließend lässt sich festhalten, dass das Angebot gemeinsam zu arbeiten mit den virtuellen Suchwerkzeugen und den physischen Such-Tokens am häufigsten wahrgenommen wurde. Zusätzlich zeigten die subjektiven Ergebnisse aus den vergleichenden Fragebögen, dass die stark realitätsbasierten physischen Such-Tokens im Vergleich zu den virtuellen Suchwerkzeugen weniger anstrengend zu bedienen sind, den Versuchspersonen mehr zusagen und als am besten geeignet für kollaboratives Recherchieren bewertet wurden. Dieses Ergebnis ist umso bemerkenswerter, da nur die Hälfte der Probanden angaben, zuvor mit (be-)greifbaren Benutzerschnittstellen in Berührung gekommen zu sein. Neben der parallelen Bedienung von Werkzeugen sind für die Kollaboration auch Aspekte wie die verbale und non-verbale Kommunikation wichtig. In der Evaluationsstudie konnte beobachtet werden, dass die Triaden je nach Werkzeug unterschiedlich aktiv (auch ,abseits' der Benutzerschnittstelle) zusammengearbeitet haben. Die Aussagen aus den Gruppeninterviews und Fragebögen bestätigen, dass die physischen Such-Tokens die Kommunikation und die aktive Beteiligung innerhalb der Triade anregten. In einer Anschlussstudie sollen diese Aspekte deshalb verstärkt untersucht werden. Hierzu ist vorgesehen die Triaden aus zwei Konföderierten und einer ,echten' Versuchsperson zusammen zu setzen. Dadurch kann die Versuchsperson gezielt mit typischen Situationen der kollaborativen Arbeit konfrontiert werden, um somit die Auswirkungen der Werkzeuge auf die Aktivität (Interaktion und Kommunikation) in einer Untersuchung besser erfassbar zu machen. 
Die in diesem Beitrag exemplarisch vorgestellten Werkzeuge zur Rechercheunterstützung zeigen auf, dass die Vermischung von realer und digitaler Interaktion Kollaborationsprozesse positiv beeinflussen kann. Die durchgeführte Studie konnte erste Erkenntnisse über mögliche Gründe liefern, die in Zukunft sowohl im Design von Benutzerschnittstellen als auch in der weiteren Forschung der kollaborativen Nutzung von Multitouch-Tischen Berücksichtigung finden sollten.

\section{Literaturverzeichnis}

Bortz, J. \& Döring, N. (2006). Forschungsmethoden und Evaluation, 4. Auflage. Heidelberg: Springer Medizin Verlag.

Gibbs, R. 2006: Embodiment and Cognitive Science. New York: Cambridge University Press.

Hoare, C. \& Sorensen, H. (2006). Enhancing Visual Information Seeking through Real-time Collaboration. Proceedings of DL-CUBA ' 06.

Ishii, H. \& Ullmer, B. (1997). Tangible Bits: Towards Seamless Interfaces between People, Bits and Atoms. Proceedings of CHI '97, pp. 234-241.

Jacob, R. J., Girouard, A., Hirshfield, L. M., Horn, M. S., Shaer, O., Solovey, E. T., Zigelbaum, J. (2008). Reality-based interaction: a framework for post-WIMP interfaces. Proceedings of CHI' 08.

Kuhlthau, C. C. (2004). Seeking meaning: a process approach to library and information services. 2nd Edition. Libraries Unlimited.

Morris, M.R., Lombardo, J., Wigdor, D. (2010). WeSearch: Supporting Collaborative Search and Sensemaking on a Tabletop Display. Proceedings of CSCW ' 10.

Perlin K. \& Fox D. Pad (1993). An Alternative Approach to the Computer Interface. Proceedings of. Computer Graphics and Interactive Techniques '93, pp. 57-64.

Tweedie, L., Spence, B., Williams, D., Bhogal, R. (1994). The attribute explorer. Proceedings of CHI '94.

Ullmer, B., Ishii, H., Jacob, R.J.K. (2003). Tangible Query Interfaces: physically constrained Tokens for manipulating Database Queries. Proceedings of INTERACT '03.

Waller, W. G. \& Kraft, D. H. (1979). A mathematical model for a weighted Boolean retrieval system. Proceedings of Information Processing and Management '79.

Weiss, M., Wagner, J., Jennings, R., Jansen, Y., Khoshabeh, R., Hollan, J. D., Borchers, J. (2009). SLAPbook Tangible Widgets on Multi-touch Tables in Groupware Environments. Proceedings of Tangible and Embedded Interaction '09.

\section{Kontaktinformationen}

Mathias Heilig, Mischa Demarmels, Katrin Allmendinger, Jens Gerken, Harald Reiterer

Universität Konstanz, Arbeitsgruppe Mensch-Computer Interaktion

Universitätsstraße 10, 78457 Konstanz

E-Mail: Vorname.Nachname@uni-konstanz.de 